\title{
ESTÁGIO CURRICULAR SUPERVISIONADO NO CONTEXTO DA COVID-19 E O DESENVOLVIMENTO PROFISSIONAL DE ESTUDANTES DE ENFERMAGEM
}

Keity Lais Siepmann Soccol ${ }^{1}$ Naiana Oliveira dos Santos ${ }^{1}$

Mara Regina Caino Teixeira Marchiori ${ }^{1}$ https://orcid.org/0000-0002-7071-3124

https://orcid.org/0000-0002-5439-2607

https://orcid.org/0000-0001-9412-7755

Objetivo: Refletir sobre as contribuições do estágio curricular supervisionado no contexto da COVID-19 para o desenvolvimento profissional dos estudantes de Enfermagem. Método: Trata-se de um estudo de reflexão desenvolvido por docentes de um curso de Enfermagem que realizam a supervisão dos estudantes durante o estágio. Resultados: O estágio curricular supervisionado no contexto da COVID-19 proporciona aos estudantes de Enfermagem a possibilidade de vivenciar experiências únicas, de desenvolver sua identidade profissional por meio da liderança, à tomada de decisão e empodera estes no que tange ao seu papel. Ainda, contribui com as ações propostas pela Campanha Nursing Now no Brasil. Conclusão: O estágio curricular supervisionado no contexto da pandemia nos serviços de Atenção Básica fortalecem a educação e o desenvolvimento profissional dos estudantes e dá visibilidade à valorização da profissão.

Descritores: Educação em Enfermagem; Estudantes de enfermagem; Infecções por coronavirus.

\section{SUPERVISED INTERNSHIP IN THE CONTEXT OF COVID-19 AND PROFESSIONAL DEVELOPMENT OF NURSING}

Objective: Reflect on the contributions of the supervised internship in the context of COVID-19 to the professional development of nursing students. Method: It is a reflection study developed by professors of a Nursing course who perform the supervision of students during the supervised internship. Results: The supervised internship in the context of COVID-19 provides nursing students with the possibility to experience unique experiences, to develop their professional identity through leadership, to decision-making and empowers students with regard to their role. It also contributes to the actions proposed by the Nursing Now Campaign in Brazil. Conclusion: The supervised internship in the context of the pandemic in primary care services strengthens students' education and professional development and gives visibility to the appreciation of the profession.

Descriptors: Education nursing; Students nursing; Coronavirus infections.

\section{PRÁCTICA SUPERVISADA EN EL CONTEXTO DE COVID Y DESARROLLO PROFESIONAL DE ESTUDIANTES DE ENFERMERÍA}

Objetivo: Reflexionar sobre las contribuciones de las pasantías supervisadas en el contexto de COVID-19 al desarrollo profesional de estudiantes de enfermería. Método: Es un estudio de reflexión desarrollado por profesores de un curso de Enfermería que realizan la supervisión de los estudiantes durante la pasantía supervisada. Resultados: La pasantía supervisada en el contexto de COVID-19 brinda a los estudiantes de enfermería la posibilidad de experimentar experiencias únicas, de desarrollar su identidad profesional a través del liderazgo, la toma de decisiones y capacita a los estudiantes con respecto a su papel. También contribuye a las acciones propuestas por la Campaña de Enfermería Ahora en Brasil. Conclusión: La pasantía supervisada en el contexto de la pandemia en los servicios de atención primaria fortalece la educación y el desarrollo profesional de los estudiantes y da visibilidad a la apreciación de la profesión.

Descriptores: Educación en enfermeira; Estudiantes de enfermería; Infecciones por coronavirus.

${ }^{1}$ Universidade Franciscana (UFN). Santa Maria, RS, Brasil.

Autor Correspondente: Keity Lais Siepmann Soccol E-mail: keitylais@hotmail.com

Recebido:12/7/20 Aceito: 03/8/20 


\section{INTRODUÇÃO}

O curso de Enfermagem em sua estrutura curricular está constituído por disciplinas que possuem atividades teóricas e práticas, que são essenciais para que o futuro profissional enfermeiro execute suas atribuições com excelência, sobretudo aquelas de gerenciamento e de liderança ${ }^{1}$. De acordo com as Diretrizes Curriculares Nacionais da Enfermagem (DCNE), a formação do enfermeiro necessita preferencialmente ocorrer nos territórios e nos serviços públicos de saúde, o que torna imprescindivel o reconhecimento da importância do Sistema Único de Saúde (SUS) nesse processo².

Dentre as disciplinas que constituem a matriz curricular do curso de Enfermagem, tem-se o Estágio Curricular Supervisionado, que confere ao futuro enfermeiro a aptidão profissional para atender as demandas e necessidades de saúde prioritárias da população, conforme a realidade epidemiológica da região e do país de acordo com as políticas públicas $^{2}$. Embora os estudantes sejam preparados para atuar em diferentes situações, a atuação em contexto de pandemias, como a do coronavirus, também denominado COVID-19, se apresenta como um novo modo de enfrentar os problemas de saúde.

A Enfermagem exerce um papel essencial no combate a COVID-19, não só por suas habilidades técnicas, mas por serem os profissionais que permanecem maior parte do tempo junto aos pacientes ${ }^{3}$, na linha de frente do cuidado. Nesse contexto, também inserem-se os estudantes de Enfermagem do último ano do curso, que estão em processo de desenvolvimento profissional e ao mesmo tempo atuando na linha de frente da COVID-19.

A profissão de Enfermagem tem ganhado visibilidade nacional e internacional por diversas organizações. Recentemente houve o lançamento da Campanha Nursing Now, em prol do incentivo da valorização do profissional da Enfermagem. A referida Campanha incentiva o empoderamento dos enfermeiros para assumir o papel de protagonistas no enfrentamento dos desafios de saúde presentes nesse século, atuando com o máximo de competência profissional e de liderança ${ }^{4}$.

A Campanha Nursing Now tem entre seus objetivos, empoderar enfermeiros para auxiliá-los a alcançar o objetivo da Cobertura Universal de Saúde, bem como, enfrentar os desafios de saúde do século XXI ${ }^{5}$. Quanto ao fortalecimento do perfil do profissional e da identidade profissional, entende-se que necessita ser estimulado ainda durante o processo de formação do enfermeiro.

A construção da identidade profissional, com a articulação da teoria à prática, influencia na atuação profissional e nas interações que são oportunizadas no decorrer do processo formativo ${ }^{6}$. Nesse sentido, atuar diante de situações im- previstas, como no enfrentamento da pandemia de COVID-19 proporciona aos estudantes de Enfermagem o empoderamento de seu papel, de exercer a liderança e os estimula a uma trajetória ao encontro do que é almejado pela Campanha Nursing Now.

Abordar o contexto da pandemia de COVID-19 e a formação em Enfermagem, sob a óptica da Campanha Nursing Now fortalece a educação e valoriza a atuação dos estudantes. Frente ao exposto, este estudo tem como objetivo refletir sobre as contribuições do Estágio Curricular Supervisionado no contexto da COVID-19 para o desenvolvimento profissional dos estudantes de enfermagem.

Trata-se de um estudo do tipo de reflexão, embasado, sobretudo na experiência de docentes na supervisão de Estágio Curricular Supervisionado de estudantes de Enfermagem que atuam no enfrentamento do COVID-19 em serviços de Atenção Básica à Saúde.

\section{O estágio curricular supervisionado no contexto da co- vid-19}

O Estágio Curricular Supervisionado oportuniza ao estudante vivenciar a prática e a realidade dos serviços de saúde, e permite o desenvolvimento do raciocínio crítico para uma atuação efetiva ${ }^{7}$. Durante o estágio, o estudante se depara com problemas e situações, para as quais se preparou no decorrer de sua formação, que compreendem desde os procedimentos técnicos até os de natureza pessoal ou emocional ${ }^{8}$.

A atuação em situações imprevisiveis e frente às adversidades, como na pandemia de COVID-19, faz parte do papel do enfermeiro. Nesse contexto, os estudantes precisam desenvolver competências e habilidades para priorizar as demandas que requerem respostas urgentes no cotidiano. As DCN instituem a liderança dentre as competências e habilidades a serem alcançadas pelo futuro enfermeiro, o que reforça a ideia de que esta deve ser incorporada a todo processo ensino-aprendizagem durante a graduação, uma vez que o líder deve ter compromisso, responsabilidade, empatia, habilidade para tomada de decisões, comunicação e o gerenciamento de forma efetiva e eficaz².

Atender a população de acordo com as necessidades de saúde é uma das propostas da Campanha Nursing Now. No entanto, para que isso seja possivel é necessário que haja a valorização da Enfermagem e também que os sistemas de saúde estejam fortalecidos para atingir a excelência do cuidado nas ações que tangem a prevenção de doenças, promoção e recuperação da saúde e a manutenção da vida das pessoas e de suas familias ${ }^{4}$.

Vivenciar o enfrentamento da pandemia durante a formação acadêmica fortalece o aprendizado diante do novo e de 
tantas informações que surgem instantaneamente. Para que os estudantes desenvolvam estratégias para solucionar os problemas e possam intervir de acordo com as suas competências no estágio, é importante que haja a integração entre ensino-serviço-comunidade, para que se possa desenvolver ações de saúde pautadas de acordo com a realidade e com as necessidades dos usuários ${ }^{1}$.

É nesse momento de adversidades causada pela pandemia que os estudantes possuem a possibilidade de exercerem com mais veemência sua liderança e atuar com enfoque na prevenção da COVID-19. Exercer a liderança nesse contexto reafirma a autonomia, a capacidade de decisão e aponta para uma prática avançada.

A possibilidade de estar no processo de trabalho enquanto acadêmico, permite aos estudantes vivenciar e planejar as ações de cuidado e assistência à saúde da população junto à equipe de saúde, além de contribuir, com as ações de educação permanente em saúde para a equipe.

A Enfermagem brasileira estava desvalorizada enquanto profissão, e no contexto da pandemia ela passa a assumir um papel de protagonista da luta contra o novo coronavírus. Ressalta-se que a Campanha Nursing Now se sobressai nesse momento de pandemia, por chamar a atenção de governos e lideranças para a valorização da Enfermagem mundial, já que estão implicados de modo direto ou indireto no cuidado das pessoas em seus diferentes ciclos de vida ${ }^{4}$.

Ao refletir sobre a valorização profissional faz-se importante lembrar a necessidade de maior investimento na força de trabalho em Enfermagem ${ }^{5}$. Nesse sentido, a mídia denuncia diariamente o descaso com a Enfermagem, o que pode ser expresso pelo quantitativo de profissionais contaminados e de óbitos por COVID-19. Os dados sobre o adoecimento dos profissionais da saúde no cenário da COVID-19 ainda são inconsistentes, mas sabe-se que eles se elevam diariamente ${ }^{3}$.

Aliado a isso, têm-se a falta de Equipamentos de Proteção Individual (EPI), jornadas de trabalho extensas, condições precárias de trabalho e a remuneração insuficiente devido à inexistência de um piso salarial. Falar sobre a formação, funções e condições de trabalho são essenciais para a debater sobre o sistema de saúde e os modos de atuação para enfrentar os surtos, epidemias e pandemias ${ }^{3}$. Nessa perspectiva, a Campanha Nursing Now, conduz as pessoas a refletir sobre a qualidade da formação 9 e a vivência dos estudantes em cenários de prática.

O Estágio Curricular Supervisionado desperta no estudante um olhar crítico reflexivo sobre a realidade que intervêm, o reconhecimento da necessidade de valorização da categoria, bem como da importância da mobilização em busca de direitos. Fatores como educação permanente, melhores oportunidades de trabalho, contingente suficiente de profissionais para um bom dimensionamento das equipes, apoio financeiro e político e o reconhecimento da liderança, são desafios para o progresso da profissão em nível global e para a cobertura universal de saúde ${ }^{10}$

Para que o enfermeiro exerça um papel de excelência é necessário que ele seja líder, criativo e inovador, motivado e que desenvolva o cuidado com ética e qualificação. Também, precisa exercer funções de gerência como planejamento e tomada de decisões ${ }^{1}$

É comum que os estudantes no estágio curricular supervisionado demonstrem insegurança e ansiedade, pois o mesmo encontra-se em um período de transição de papéis, que vai da saída da vida acadêmica à inserção no mercado de trabalho'. Por isso, a necessidade de fortalecer a educação e o desenvolvimento profissional, já que são essenciais para a valorização da profissão e para a transformação social.

O compromisso com a formação presencial nas atividades de estágio inclui o reconhecimento das condições laborais e os riscos biológicos, físicos e psicossociais a que estão expostos os estudantes, bem como a sobrecarga que o estágio pode acarretar. A atividade do estágio deve proporcionar condições adequadas aos estudantes, que não os exponham ao risco de aquisição da doença e a iminência do óbito. Para isso, deve-se garantir materiais, insumos e EPI; além dos professores avaliavam criticamente o cenário do estágio, se as condições dos serviços de saúde estão adequadas para a formação e segurança do estudante. Os professores supervisores do Estágio Curricular Supervisionado devem acompanhar as práticas do estudante, para garantir a segurança e fornecê-los apoio na construção de práticas e saberes, com base numa aprendizagem significativa.

Aprender com as experiências da pandemia atual é fundamental para a elaboração de estratégias de enfrentamento desta e de outras que poderão surgir no futuro ${ }^{3}$. Destaca-se que o processo de aprendizado em liderança demanda participação na tomada de decisão, a busca de ideias inovadoras e novas experiências ${ }^{11}$.

Discutir e proporcionar momentos de reflexão contribuiu para o fortalecimento da educação e para o incentivo de uma formação em que os estudantes em um contexto de pandemia atinjam ao máximo suas competências e habilidades.

\section{LIMITAÇÕES DO ESTUDO}

A proposta do estudo é provocar reflexões acerca do tema, mais especificamente em relação ao Estágio Curricular Supervisionado no contexto de COVID-19 e desenvolvimento profissional de estudantes de enfermagem. Elenca-se como limitações do estudo, a produção científica ainda escassa so- 
bre essa temática, o que dificulta a possibilidade de corroborar com as reflexões do presente estudo.

\section{Contribuições do Estudo para a Prática}

Tendo em vista a escassez e relevância de pesquisas que abordem essa temática, esse relato pode contribuir tanto com os processos formativos, quanto na logística do Estágio Curricular Supervisionado no contexto de COVID-19 para o direcionamento sistematizado e, acompanhamento dos estudantes nos serviços de saúde. Portanto, sendo esse contexto emergente, a comunicação ensino-serviço-comunidade se torna fundamental para a formação de enfermeiros com competências e habilidades para trabalharem no contexto de enfrentamento de uma pandemia.

\section{CONCLUSÃO}

O Estágio Curricular Supervisionado nos serviços de saúde no contexto da pandemia contribui para o fortalecimento da educação e para o desenvolvimento profissional dos estudantes. Nesse sentido, impulsiona as ações propostas pela Campanha Nursing Now em prol do reconhecimento e valorização da profissão.

O cenário inédito de COVID-19 permite ao estudante desenvolver uma visão e um pensamento crítico acerca da realidade e instiga para uma transformação social. Além disso, pro- porciona novas experiências e aprendizados únicos, em que os estudantes têm a oportunidade de desenvolver a liderança, o protagonismo e o empoderamento do seu papel. Ser professor supervisor demanda despertar o pensamento do estudante para a importância da profissão e fortalecer a educação.

Os desafios da atuação, as dificuldades da organização da gestão e escassez de recursos materiais intensifica a reflexão sobre as necessidades de melhorias no processo de trabalho e na valorização dos profissionais de enfermagem.

A possibilidade de realizar Estágio Curricular Supervisionado no atual contexto do país, revela a importância dos estudantes de Enfermagem no enfrentamento a COVID-19, na prevenção à doença e incita a valorização da profissão.

Contribuições dos Autores: Keity Laís Siepmann Soccol: a) concepção do estudo; b) interpretação dos dados; c) redação e/ou revisão crítica do manuscrito; d) aprovação da versão final a ser publicada. Naiana Oliveira dos Santos: a) concepção do estudo; b) interpretação dos dados; c) redação e/ ou revisão crítica do manuscrito; d) aprovação da versão final a ser publicada. Mara Regina Caino Teixeira Marchiori: a) concepção do estudo; b) interpretação dos dados; c) redação e/ ou revisão crítica do manuscrito; d) aprovação da versão final a ser publicada.

\section{REFERÊNCIAS}

1. Berghetti L, Franciscatto LHG, Getelina CO. Formação do enfermeiro acerca do gerenciamento: entraves e perspectivas. Rev Enferm Cent-Oeste Min. [Internet]. 2019 [cited 2020 Jul 21]; 9(e). Available from: https://doi.org/10.19175/recom. v19i0.2820.

2. Ministério da Educação (BR). Resolução no 573, de 31 de janeiro de 2018. Diretrizes Curriculares Nacionais (DCN) para o curso de graduação Bacharelado em Enfermagem. Brasília: MEC; 2001. [Internet]. 2001 [cited 2020 Jul 21]. Available from: http://www.in.gov.br/materia/-/asset_publisher/ Kujrw0TZC2Mb/content/id/48743098/dol-2018-11-06resolucao-n-573-de-31-de.

3. Souza e Souza LPS, Souza AG. Enfermagem brasileira na linha de frente contra o novo Coronavirus: quem cuidará de quem cuida? J. nurs. health. [Internet]. 2020 [cited $2020 \mathrm{Jul}$ 21];10(e):20104005. Available from: http://dx.doi.org/10.15210/ jonah.v10i4.18444.

4. Mendes IAC. Agora, sim!!! Lançamento da Campanha Nursing Now Brasil. Enferm Foco [Internet]. 2019 [cited 2020 Jul 21]; 10(2). Available from: https://doi.org/10.21675/2357707X.2019.v10.n2.2331.

5. Kennedy A. Wherever in the world you find nurses, you will find leaders. Rev. Latino-Am. Enfermagem. [Internet]. 2019 [cited 2020 Jul 21]; 27(e). Available from: https://doi. org/10.1590/1518-8345.0000.3181.

6. Silva GO, Souza PM, Batista AN, Barbosa CDM, Barreto IS, Ribeiro LCM. Estágio Curricular Supervisionado em autarquia profissional: contribuições para a formação em Enfermagem. Enferm Foco [Internet]. 2019 [cited 2020 Jul 21];10(6):205211. Available from: http://revista.cofen.gov.br/index.php/ enfermagem/article/view/2763/673.

7. Souza DJ, Faria MF, Cardoso RJ, Contim D. Estágio curricular supervisionado sob a óptica

dos enfermeiros supervisores. Rev. enferm. atenção saúde [Internet]. 2017 [cited 2020 Jul 21];6(1):39-51. Available from: http://seer.uftm.edu.br/revistaeletronica/index.php/enfer/ article/view/1677/pdf

8. Restelatto MTR, Dallacosta FM. Vivências do acadêmico de enfermagem durante $\circ$ estágio com supervisão indireta. Enferm Foco [Internet]. 2018 [cited 2020 Jul 21]; 9(4):34-38. Available from: http://revista.cofen.gov. br/index. php/enfermagem/article/view/l156.

9. Marziale MHP. Reflexões sobre a produção e a divulgação do conhecimento científico da Enfermagem. Advances in Nursing [Internet]. 2019 [cited 2020 Jul 21];1(1):4-7. Available from: http://dx.doi.org/10.5433/anh.2019vl.id38062.

10. Cassiani SHB, Lira Neto JCG. Nursing perspectives and the "Nursing Now" campaign. Rev Bras Enferm. [Internet]. 2018 [cited 2020 Jul 21];71(5):2351-2. Available from: https://doi. org/10.1590/0034-7167.2018710501.

11. Oliveira C, Santos LC, Andrade J, Domingos TS, Spiri WC. A liderança na perspectiva de enfermeiros da Estratégia Saúde da Familia. Rev Gaúcha Enferm. [Internet]. 2020 [cited 2020 Jul 21]:4l(e). Available from: https://doi.org/10.1590/19831447.2020.20190106. 